\title{
Analysis and measurement of damping characteristics of linear generator
}

\author{
Takahito Adachi ${ }^{\mathrm{a}, *}$ and Kenji Takahara ${ }^{\mathrm{b}}$ \\ ${ }^{a}$ Department of Mechanical and Systems, Houkoku Gakuen High School, Kitakyusyu, Fukuoka, Japan \\ ${ }^{\mathrm{b}}$ Department of Electrical Engineering, Faculty of Engineering, Fukuoka Institute of Technology, \\ Fukuoka, Japan
}

\begin{abstract}
The purpose of this study is to analyse and to measure damping characteristics of a linear generator. The linear generator can convert any mechanical vibration to electric power. The damping characteristics of the linear generator change due to the changes of the connected resistance. Therefore, a linear generator can be used as a controllable damper. The viscous damping coefficients were calculated by a finite element method. The prototype linear generator was produced based on the simulation and its damping characteristics were measured. It was confirmed that the viscous damping coefficient was controlled by PWM signal. Furthermore, the charging characteristics were measured when it mover was driven by constant vibration of amplitude $30[\mathrm{~mm}]$ and frequency $3[\mathrm{~Hz}]$. It was conceivable that the proposed linear generator can be used as a generator or a controllable damper as necessary.
\end{abstract}

Keywords: Linear generator, PWM signal, viscous damping coefficient, semi-active suspension

\section{Introduction}

As a dispatch reason of Japan Automobile Federation of 2014, there were the most relief requests about the over discharge of the battery [1]. By the electricity consumption increase in electric components in the car, the number of dispatch is increasing yearly. Therefore, a system to cut the electric power consumption and to manage the electricity effectively is required.

Vibration during a vehicle is absorbed by the suspension and shock absorbers, and converted to heat, in order to give good riding quality and/or protect in-car goods. If this vibration energy is converted to electricity energy, it is conceivable that one of the efficient energy applications of electric vehicle will be achieved. Suganuma et al. suggested an electromagnetic damper system using a ball screw and a DC motor to control vibration of a vehicle and to generate electric power from the vibration as needed [2]. Because the vibration is converted into rotary motion by a gear in the system, the structure is complicated. On the other hand, linear generators which can convert vibration into electric energy directly as linear motion have been proposed [3]. The fundamental structure of a linear generator is classified broadly into two types; the linear actuated magnet type and the linear actuated plunger type. Wang et al. designed a special spring and experimentally produced a small linear generator in order to utilize

\footnotetext{
${ }^{*}$ Corresponding author: Takahito Adachi, Department of Mechanical and Systems, Houkoku Gakuen High School, Yanagimachi 4-5-1, Moji-ku, Kitakyusyu, Fukuoka 800-0025, Japan. Tel.: +81 093371 3037; Fax: +81 093 371 3032; E-mail: ad16102@bene.fit.ac.jp.
}

1383-5416/16/\$35.00 (c) 2016 - IOS Press and the authors. All rights reserved

This article is published online with Open Access and distributed under the terms of the Creative Commons Attribution NonCommercial License (CC BY-NC 4.0). 


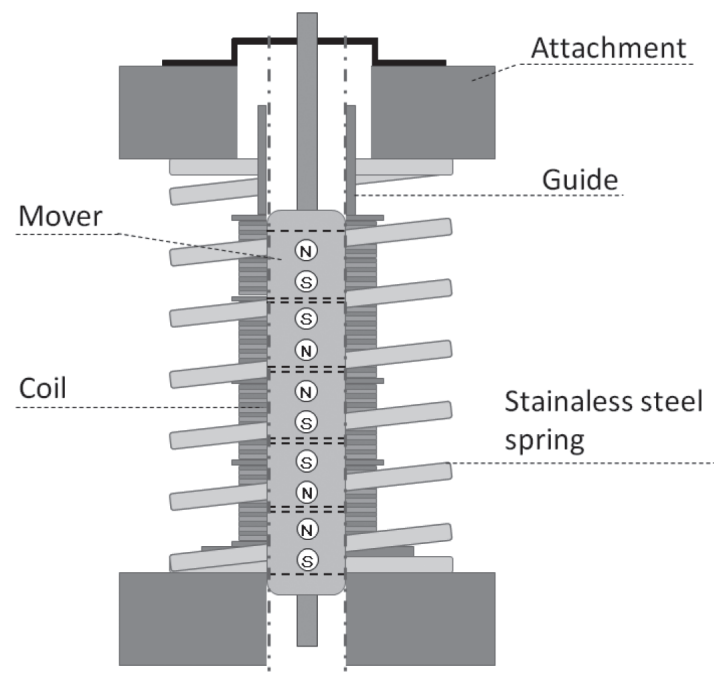

Fig. 1. Fundamental structure of linear generator.

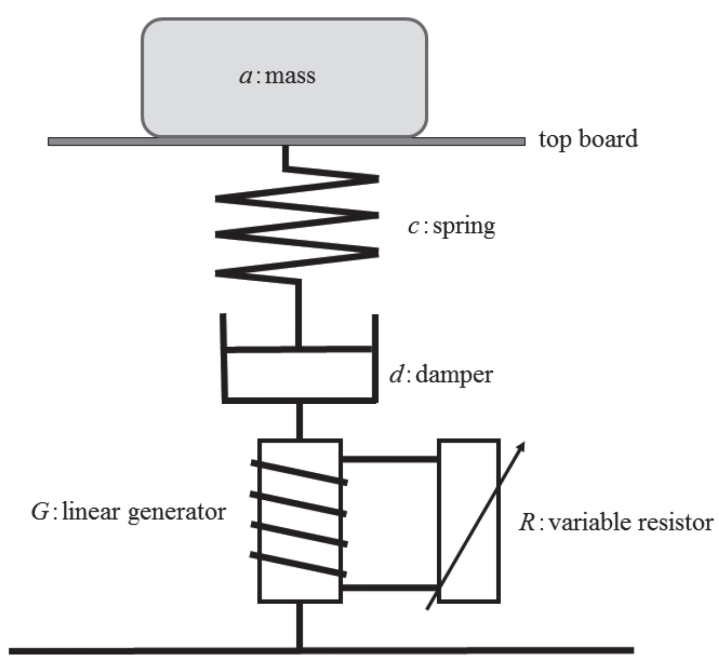

Fig. 2. Analytical model.

vibration of a moving object [4]. Cawthorne et al. proposed a permanent-magnetic generator driven by an engine [5]. Though low-electric-powered generators of tens of $\mathrm{mW}$ are also proposed for driving ICs, their driven frequencies are comparatively higher than that of the vibration of automobile. A permanent-magnetic type linear generator has been designed in order to utilize vibration of automobile as regenerative electric power by Takahara et al. [6]. The electric current through the linear generator changes by resistance connected with. The damping force of mover changes according to the magnetic field induced by the electric current. The linear generator would be able to be used as a semi-active suspension system which is controlled by changing impedance connected to the generator.

Here, the linear generator developed by Takahara et al. will be modified and its damping characteristics will be simulated and measured. In this paper, the fundamental structure of the linear generator will be described first. Subsequently, the damping characteristics of the modified linear generator will be calculated by finite element method (FEM). The linear generators will be produced on the basis of the simulation. The damping effect of the produced linear generator will be tested by examinations. Furthermore, the charging characteristics will be measured when it mover was driven by constant vibration.

\section{Materials and method}

\subsection{Simulation of characteristics of linear generator}

Figure 1 shows the structure of the linear generator. The mover is made using $\mathrm{Nd} \mathrm{Fe}-\mathrm{B}$ magnets, which are placed so that the same magnetic poles face each other. The coils are placed in the stator with the same interval as the magnets. Alternate coils connected in series were wound in opposite directions to the next coil, so that the generated power synchronizes and becomes large.

The dynamic characteristics of the linear generator are analyzed by dynamic interaction analysis which combined electromagnetic field analysis with structure analysis using FEM software ANSYS (CYBERNET SYSTEMS Co. Ltd.). The analytical model that a spring and a damper are connected to the linear generator is shown as Fig. 2. 


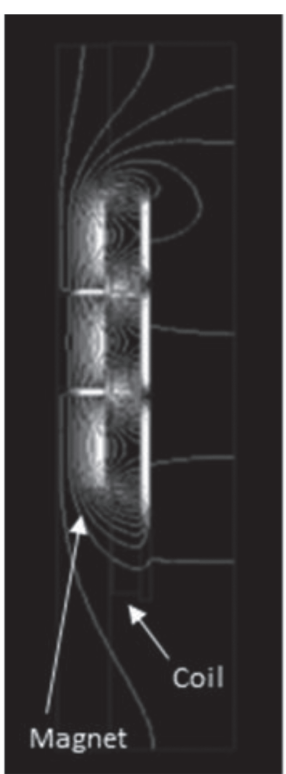

(a) Start position

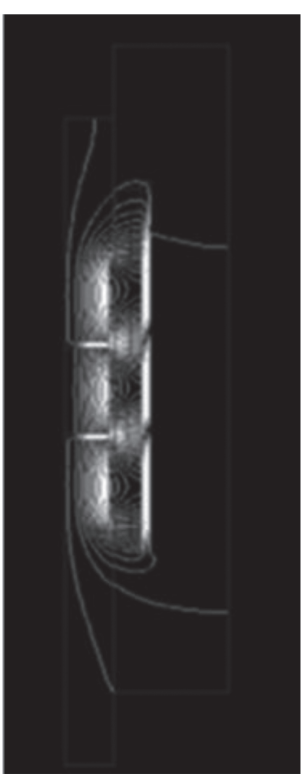

(b) Stop position

Fig. 3. The calculated magnetic configuration.

The calculated magnetic configuration is shown in Fig. 3. In this figure (a) and (b), three left-sided rectangles are magnets, and four right-sided rectangles are coils. The (a) and (b) is the magnetic configuration at start position and stop position, respectively.

The mover is driven by step load with step size of $3.3[\mathrm{~ms}]$. The displacement of the mover is determined by architectural analysis. The load current and the induction magnetic field are calculated by the displacement of the mover by electromagnetic field analysis. Because the motion of the mover is impeded by the force produced by the induction magnetic field, the displacement of the mover is afresh calculated by structure analysis by adding the step load including the increased force. The series of calculations are repeated until the displacement of the mover converges.

For the mounting to a minicar, the linear generator was designed at $60[\mathrm{~mm}]$ in diameters and at $220[\mathrm{~mm}]$ in lengths. Button type Nd Fe-B magnets are used in the mover. The height of the button type $\mathrm{Nd} \mathrm{Fe}-\mathrm{B}$ magnet used is $115[\mathrm{~mm}]$ and its diameter is $25[\mathrm{~mm}]$ (the coercive force: $1350000[\mathrm{~A} / \mathrm{m}]$ ). One coil is wound using conductive wire from $28.5[\mathrm{~mm}]$ of the inner diameter to $60[\mathrm{~mm}]$ of the outer diameter. The filling rate is assumed as 56 [\%]. The electric resistivity of the conducting wire is assumed as $0.17[\mathrm{p} \Omega \mathrm{m}]$. The spring constant is chosen as $6.211[\mathrm{~N} / \mathrm{m}]$ so that the change of the length of the spring is $30[\mathrm{~mm}]$ when the load of $15[\mathrm{~kg}]$ is applied.

By calculating the electric current for various resistances connected to the linear generator, the displacement of the mover is determined. Figures 4(a) and (b) illustrate the displacements of the mover when the output terminal of the linear generator is opened and is shorted, respectively. The viscous damping coefficient is acquired from the calculated displacement by the following Eq. (1). Here, $A_{0}$ and $A_{n}$ are the first and last amplitude value, respectively. The change of the viscous damping coefficient for logarithmic resistance connected to the linear generator is shown in Fig. 5. When a vehicle vibrates at $0.3[\mathrm{~m} / \mathrm{s}]$, it is confirmed that the damping force of $0.034[\mathrm{kN}]$ is provided as the maximum. This means that the linear generator increases up to about $7[\%]$ of damping force of a regular damper $(0.5[\mathrm{kN}], 0.3$ 


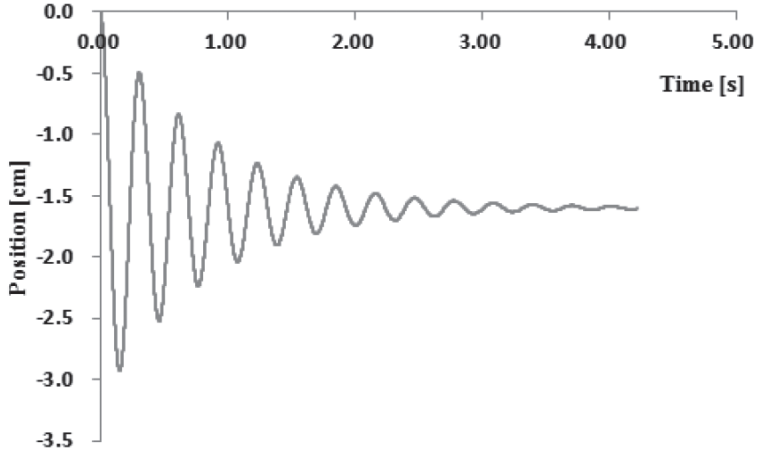

(a) Opened circuit

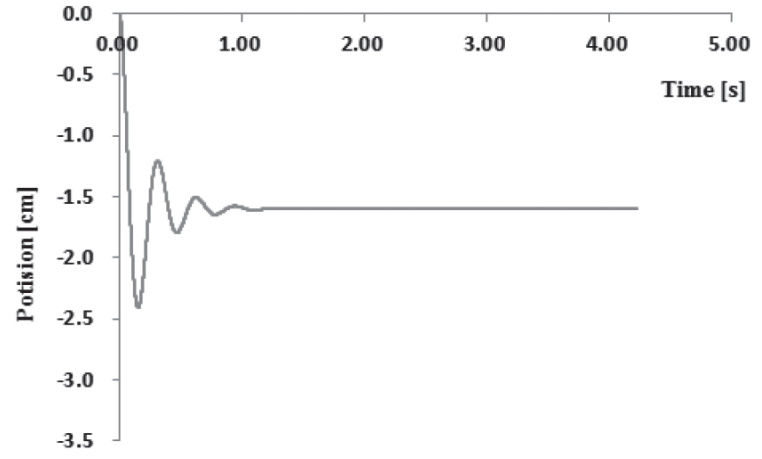

(b) Shorted circuit

Fig. 4. Displacements of the mover.

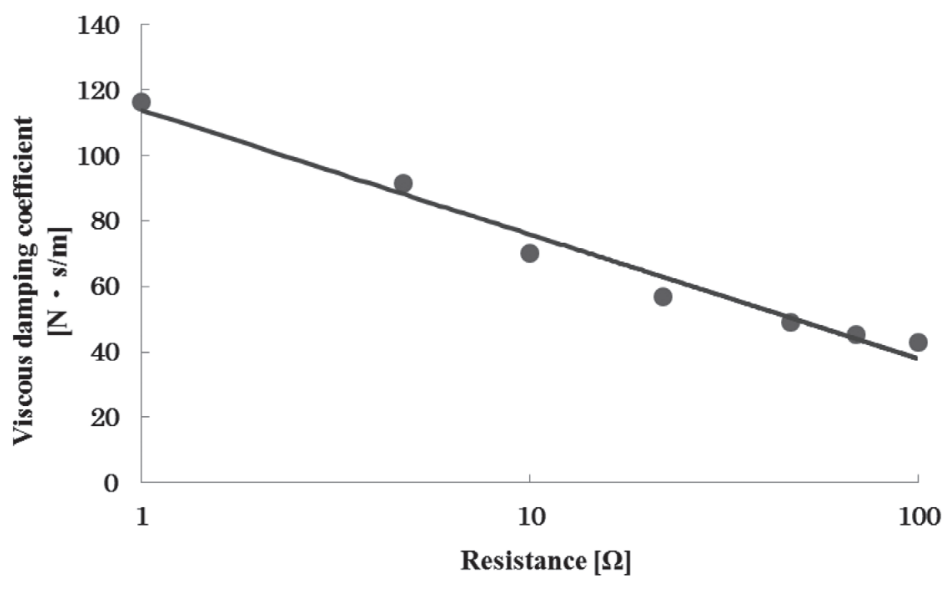

Fig. 5. Viscous damping coefficient for resistance.

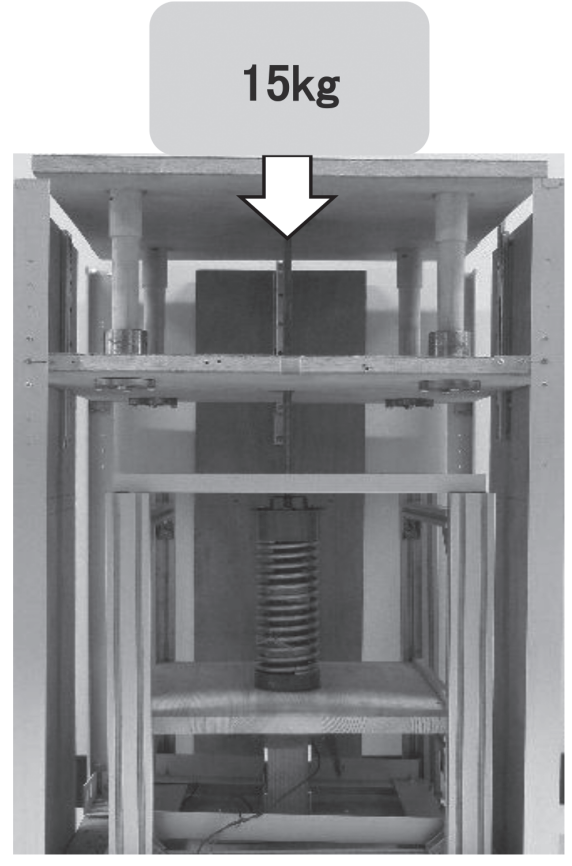

Fig. 6. Experimental equipment.

$[\mathrm{m} / \mathrm{s}])$ of the minicar.

$$
\zeta=-\left\{\frac{\log _{e}\left(\frac{A_{n}}{A_{0}}\right)}{2 \pi n}\right\}
$$

\subsection{Damping characteristics}

The prototype of the linear generator is produced based on the simulation. As a phase stator, conductive wire of $0.6[\mathrm{~mm}]$ in diameter is wound 420 times. Figure 6 shows the experimental equipment. 


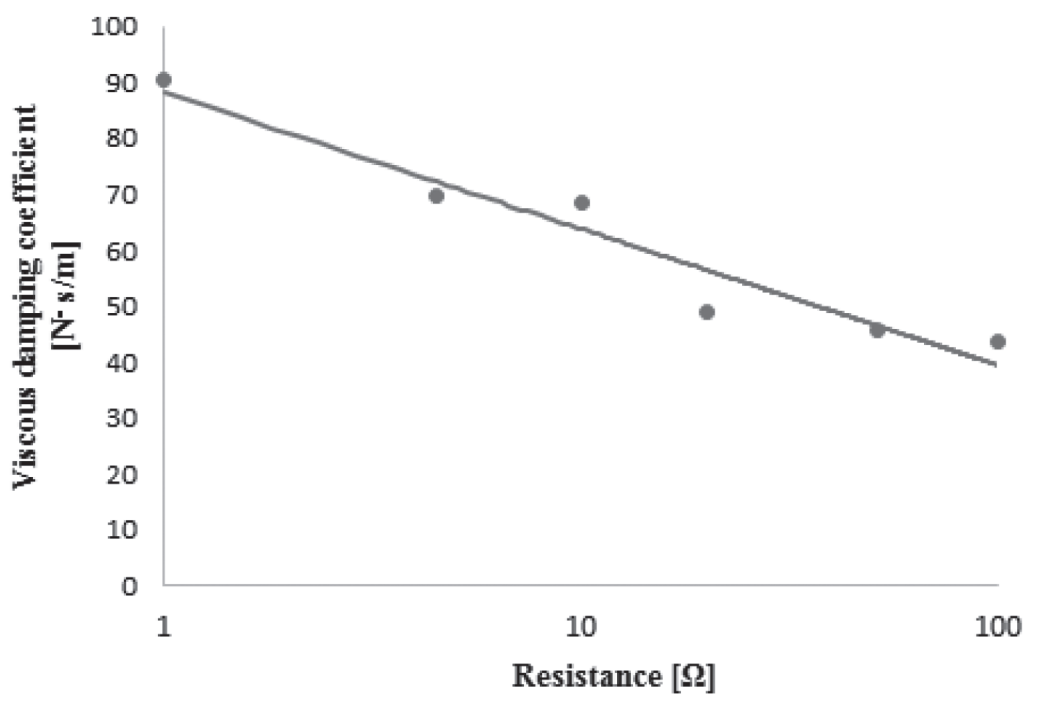

Fig. 7. Viscous damping coefficient for resistance.

Vibration characteristics of the produced linear generator is measured for changes in a resistance value from $1[\Omega]$ to $100[\Omega]$ in experimental settings same to the above simulation. When the circuit is opened the real vibration resembles the simulation result closely. But, the vibration continues for a little longer than the simulation result when the circuit is shorted.

When the resistance of $1[\Omega]$ is connected, the measured viscous damping coefficient is $90.40[\mathrm{Ns} / \mathrm{m}]$. The measured viscous damping coefficient for logarithmic resistance connected to the linear generator is shown in Fig. 7. It is considered that because the internal resistance of the generator is $200[\Omega]$ and large, the electric current does not flow enough even when a low resistance is connected to the generator.

The above experiment shows that the linear generator can be used as a damper which has a variable damping coefficient. An experimental system is constructed as Fig. 8, to regulate a viscous damping coefficient electronically.

The maximum output voltage of the linear generator is about $6[\mathrm{~V}]$ and it is small value. Therefore, the electric current through the connected resistance is small because the commutated output voltage is a smaller value. That is, it is necessary that the voltage drop in the circuit is small as much as possible, in order to get the damping force large. Thus, a relay is used instead of a FET as a switching device.

A small electromagnetic relay (G6C-2117P-US, Omron) and resistance of $0.5[\Omega]$ are connected to the linear generator. The electromagnetic relay is driven with PWM signal of $8[\mathrm{~Hz}]$. The equivalent resistance value connected to the linear generator is calculated from Eq. (2) in analogy with the conversion of the resistance value through the DC-DC converter. Here, $\mathrm{d}$ is the duty ratio of PWM signal. The change of the equivalent resistance value for the change of the duty ratio is shown in Fig. 9.

$$
R_{e q}=\frac{1}{d^{2}} R_{L}
$$

Figure 10 shows the viscous damping coefficient for the duty ratio of PWM. This figure shows that the viscous damping coefficient is linearly changed from $32.49[\mathrm{Ns} / \mathrm{m}]$ to $102.22[\mathrm{Ns} / \mathrm{m}]$ by changing the duty ratio of PWM signal. When vibration of $0.3[\mathrm{~m} / \mathrm{s}]$ is added to the linear generator, a damping force of up to $30.6[\mathrm{~N}]$ is obtained. Therefore, it is thought that the relay is not switched appropriately at large duty ratio. 


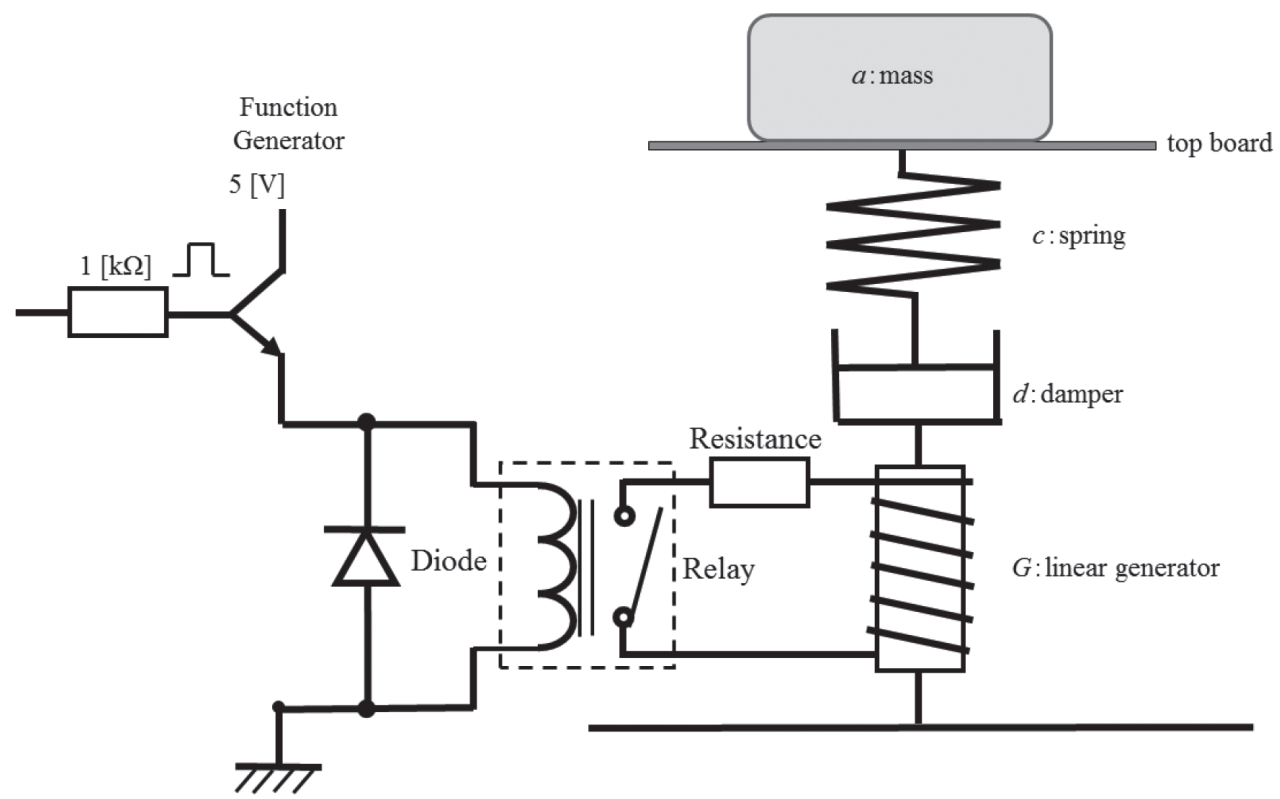

Fig. 8. Outline of the experimental circuit.

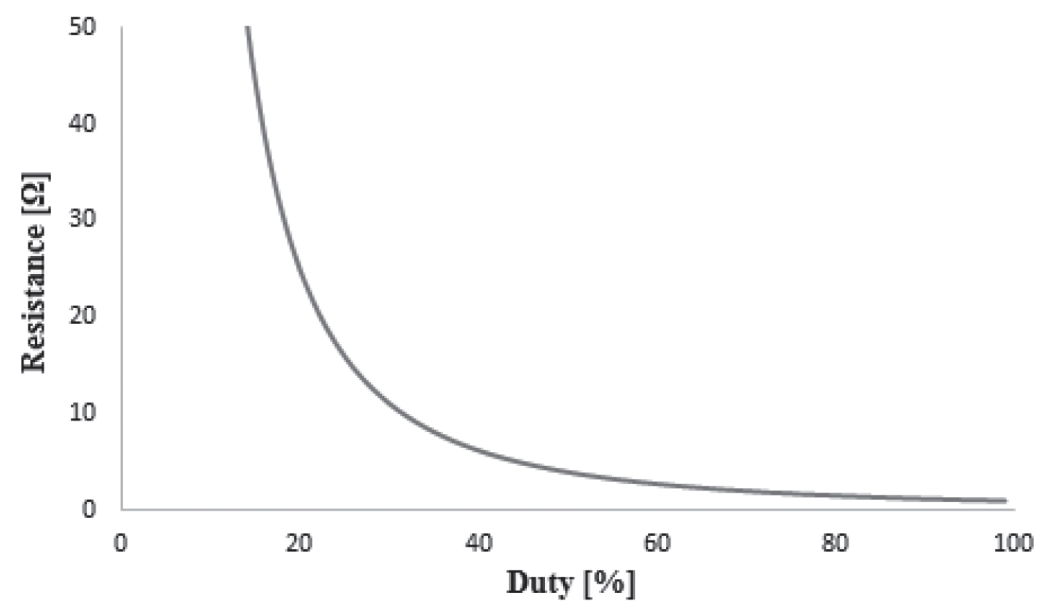

Fig. 9. Equivalent resistance value for the duty ratio.

For a comparison, the duty ratio of the PWM signal is converted into a resistance value connected to the linear generator. For example, here, when the relay is driven by 95 [\%] of duty ratio, resistance of $1[\Omega]$ corresponds approximately to being connected to the linear generator. The viscous damping coefficient for the resistance of $1[\Omega]$ is $90.40[\mathrm{Ns} / \mathrm{m}]$, and it for $95[\%]$ of duty ratio is $96.32[\mathrm{Ns} / \mathrm{m}]$. Furthermore, the viscous damping coefficient for the resistance of $100[\Omega]$ is $43.80[\mathrm{Ns} / \mathrm{m}]$, and it for $15[\%]$ of duty ratio is 43.57 [Ns/m]. It is considered that the effect of changing PWM ratio is comparable to the damping effect by changing the resistance. Electric power of $60[\mathrm{~mW}]$ at a maximum is required for driving the relay. It may need very small electric consumption to operate as a semi-active damper.

Therefore, it is thought that this system would be useful to realize the inexpensive semi-active damper 


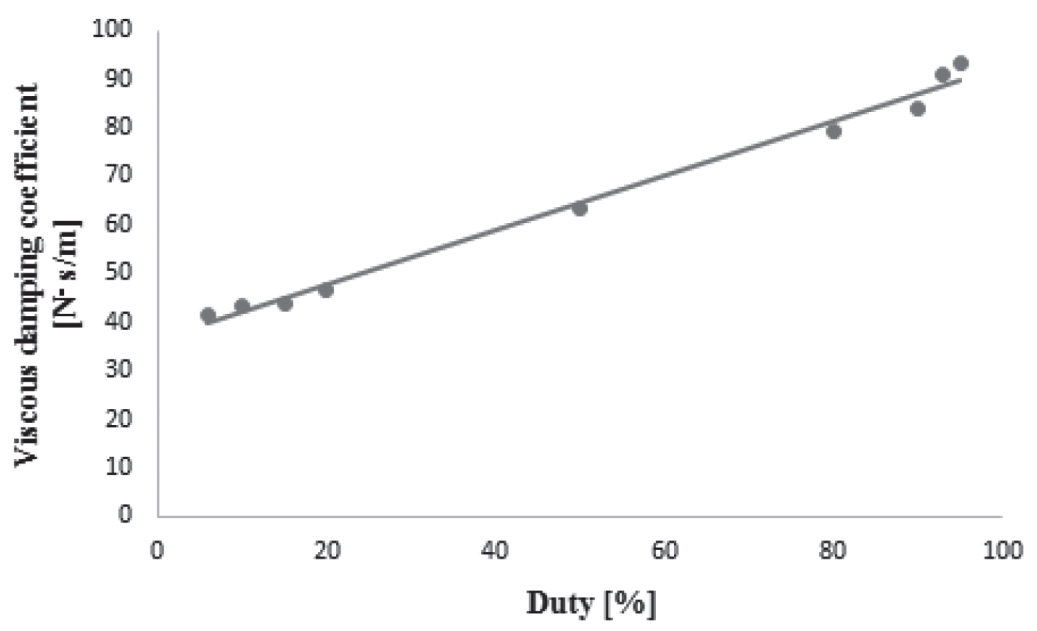

Fig. 10. Viscous damping coefficient for duty ratio of PWM signal.

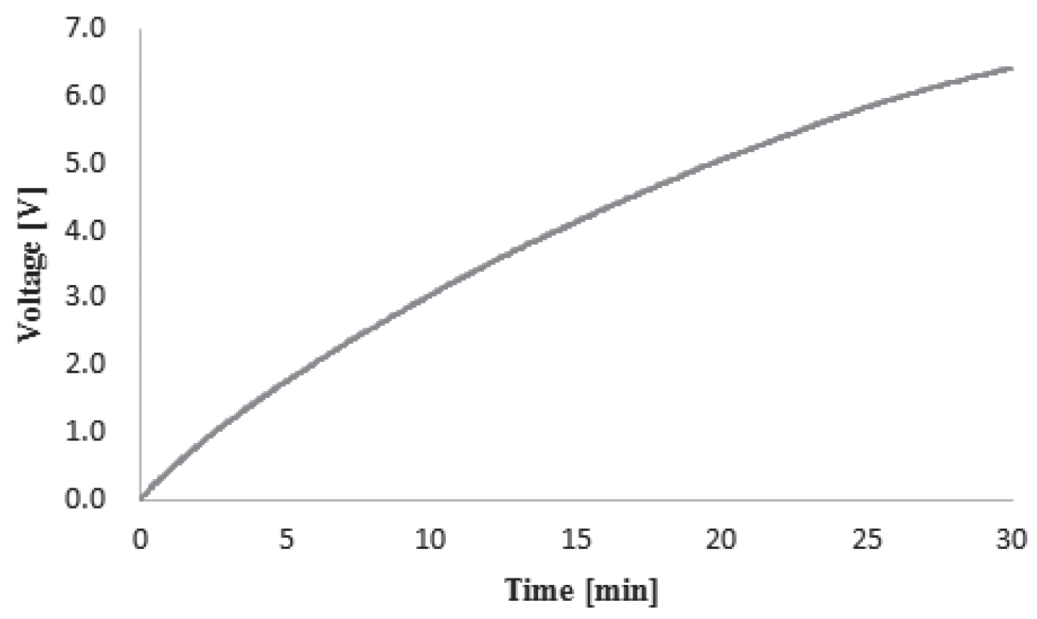

Fig. 11. Change of charge voltage of electric double-layer capacitor.

system which is driven by small electricity.

\subsection{Generation characteristics}

The proposed linear generator will be installed in a suspension in order to effectively convert vibration of a minicar to electric energy. Then the charging characteristics to the electric double-layer capacitor of $5[\mathrm{~F}]$ is measured when the output voltage is provided through a quadruple-voltage rectifier circuit. The mover is driven by constant vibration of amplitude $30[\mathrm{~mm}]$ and frequency $3[\mathrm{~Hz}]$. Here, the amplitude and the frequency are standard vibration conditions of the running vehicles. Figure 11 illustrates the change in the charging voltage to the electric double-layer capacitor.

It is possible to charge up to $6[\mathrm{~V}]$ in about 25 [min]. By the influence of large capacity and the voltage drop in the rectifier circuit, the charge voltage rises slowly. The average output power is about $60[\mathrm{~mW}]$ even when the mover is driven at low frequency. It is considered that the energy can be used as a power supply for nightlight or in an emergency if vibrational energy during drive charges up a capacitor. 
The average electric-generating capacity covers the maximum power for driving the relay. Therefore, the proposed linear generator can be used for charging electric power converted vibration. Furthermore, it may be also used as a controllable damper as necessary.

\section{Conclusion}

In this paper, vibration characteristics of the linear generator to be integrated in a minicar was simulated by dynamic interaction analysis of FEM. The linear generator was produced based on the simulation, and its damping characteristics was measured. It was confirmed that the damping characteristics of the linear generator was regulated by the PWM signal like by changing the resistance connected to it. That is, changing the duty ratio of the PWM signal is equivalent to changing resistance value. That is, the proposed linear generator would be able to be useful for a controllable damper system. On the other hand, it is confirmed that the linear generator can be convert the vibration to electric power and charge the capacitor when the mover of the proposed linear generator is driven at low frequency. Therefore, the proposed linear generator can be used as a generator or a controllable damper as necessary.

Here, because the linear generator was experimentally produced small for a minicar, its output power was small. This is the result of downsizing of the linear generator to mount in a minicar. In order to produce a high-power and small generator, it is needed to be redesigned optimally.

Furthermore, the proposed linear generator may be applied as a vibration absorption ingredient for houses $[7,8]$. It would store the small vibration of the house as electricity, and might control the large vibration as a damper using the stored electricity.

\section{References}

[1] Japan Automobile Federation, Road service based on data, http://www.jaf.or.jp/rservice/data/ 2014/year.htm, 2015.

[2] A. Suganuma and K. Nakano, Electromagnetic Damper Control Development with Consideration for Energy Regeneration, 2011 National Convention Record IEE Japan, No. 4, 2010, pp. 310-311.

[3] I. Boldea and S.A. Nasar, Linear electric actuators and generators, IEEE Trans. Energy Conversion 14(3) (1999), $712-717$.

[4] J. Wang, W. Wang, G.W. Jewell and D. Howe, Design and experimental characterization of a linear reciprocating, IEE Proc-Electr. Power Appl. 145(6) (1998), 509-518.

[5] W.R. Cawthorne, P. Famouri, J. Chen, N.N. Clark, T.I. McDaniel, R.J. Atkinson, S. Nandkumar, C.M. Atkinson and S. Petreanu, Development of a linear alternator-engine for hybrid electric vehicle applications, IEEE Trans. Vehicular Thecnology 48(6) (1999), 1797-1802.

[6] K. Takahara et al, Characteristic analysis and trial manufacture of permanent-magnetic type linear generator, IEEJ Trans. IA 127(6) (2007), 669-67.

[7] Y. Aoyama, H. Fujitani and Y. Mukai, Proposal of semi-active control with variable rotational Inertia force Damper in base-isolated layer, Architectural Institute of Japan, Summaries of technical papers of annual meeting, 2013, 401-402.

[8] K. Hiroishi and H. Fujitani, Earthquake resistant performance evaluation by "Is" of retrofitted buildings by high damping visco elastic dampers, Architectural Institute of Japan, Summaries of technical papers of annual meeting, B-2, 2011, 803-804. 